\title{
An optimizing reduced order FDS for the tropical Pacific Ocean reduced gravity model
}

\author{
Zhendong $\mathrm{Luo}^{a, \dagger}$, Jing Chen ${ }^{b, \ddagger}$, Jiang $\mathrm{Zhu}^{c, \S}$, \\ Ruiwen Wang ${ }^{c, \boldsymbol{I}}$, and I. M. Navon ${ }^{d, *, \downarrow}$ \\ ${ }^{a}$ School of Science, Beijing Jiaotong University, Beijing 100044, P. R. China \\ ${ }^{b}$ College of Science, China Agricultural University, Beijing 100083, P. R. China \\ ${ }^{c}$ Institute of Atmospheric Physics, Chinese Academy of Sciences, Beijing 100029, P. R. China \\ ${ }^{d}$ School of Computational Science and Department of Mathematics, Florida State University, Dirac Sci. \\ Lib. Bldg., \#483, Tallahassee, FL 32306-4120, USA
}

\section{SUMMARY}

Proper orthogonal decomposition (POD) and singular value decomposition (SVD) methods are used to study a finite difference discretization scheme (FDS) for the tropical Pacific Ocean reduced gravity model. Ensembles of data are compiled from transient solutions computed from the discrete equation system derived by FDS for the tropical Pacific Ocean reduced gravity model. The optimal orthogonal bases are used to reconstruct the elements of the ensemble with POD and SVD. Combining the above approach with a Galerkin projection procedure yields a new optimizing FDS model of lower dimensions and high accuracy for the tropical Pacific Ocean reduced gravity model. An error estimate of the new reduced order optimizing FDS model is then derived. Numerical examples are presented illustrating that the error between the POD approximate solution and the full FDS solution is consistent with previously obtained theoretical results, thus validating the feasibility and efficiency of POD method.

Received August 28, 2006;

KEY WORDS: proper orthogonal decomposition; singular value decomposition; finite difference scheme; the tropical Pacific Ocean reduced gravity model

* Correspondence to: I. M. Navon, School of Computational Science and Department of Mathematics, Florida State University, Dirac Sci. Lib. Bldg., \#483, Tallahassee, FL 32306-4120, USA

† E-mail: zhdluo@bjtu.edu.cn

‡ E-mail: jing_quchen@163.com

$\S$ E-mail: jzhu@mail.iap.ac.cn

ฯ E-mail: wangrw@mail.iap.ac.cn

$\diamond$ E-mail: navon@scs.fsu.edu

Contract/grant sponsor: the National Science Foundation of China; Grant/grant numbers: 10471100, 40437017, and 60573158

Contract/grant sponsor: Beijing Jiaotong University Science Technology Foundation; Grant/grant number: SJ06000 


\section{INTRODUCTION}

The finite difference scheme (FDS) is one of the important discretization approaches for numerical solution of a system of partial differential equations, for example those used for the tropical Pacific Ocean reduced gravity model. However, since the tropical Pacific Ocean reduced gravity model is used to compute the variability of fluid flow and fluid total layer thickness over total field near by tropical Pacific Ocean (see [1], or [2]), its high resolution FDS is characterized by a large number of degrees of freedom. Thus, an important problem is how to alleviate the computational load and save time-consuming calculations and resource demands in the computational process in a way that guarantees a sufficiently accurate numerical solution. Proper orthogonal decomposition (POD), also known as the Karhounen-Loève expansion in signal analysis and pattern recognition (see [3]), or principal component analysis in statistics (see [4]), or the method of empirical orthogonal functions in geophysical fluid dynamics (see [5], [6]) or meteorology (see [7]), is a technique offering an adequate approximation for representing fluid flow with a reduced number of degrees of freedom, i.e., with a lower dimensional model (see [8]) so as to alleviate the computational load and provide $\mathrm{CPU}$ and memory requirements savings. The POD method mainly provides a useful tool for efficiently approximating a large amount of data. The method provides an orthogonal basis for representing the given data in a certain least squares optimal sense, that is, it provides a way to find optimal lower dimensional approximations of the given data. In addition to being optimal in a least squares sense, POD has the property that it uses a model decomposition that is completely data dependent and does not assume any prior knowledge of the process employed to generate the data. This property is advantageous in situations where a priori knowledge of the underlying process is insufficient to warrant a particular choice of basis. Combined with the Galerkin projection procedure, POD provides a powerful method for generating lower dimensional models of dynamical systems that have a very large or even infinite dimensional phase space. The fact that this method always searches for linear (or affine) subspaces instead of curved submanifolds renders it computationally tractable. In many cases, the behavior of a dynamic system is governed by characteristics or related structures, even though the ensemble is formed by a large number of different temporal solutions.

Since the POD method can capture these temporal and spatial structures by applying a statistical analysis to the ensemble of data, it has found widespread applications in statistics. In fluid dynamics, Lumley first employed the POD technique to capture the large eddy coherent structures in a turbulent boundary layer (see [9]); this technique was further extended in [10], where a link between the turbulent structure and the dynamics of a chaotic system was investigated. In Holmes et al. [9], the overall properties of POD were reviewed and extended to widen the applicability of the method. The method of snapshots was introduced by Sirovich [11], and is widely used in applications to reduce 
the order of the POD eigenvalue problem. Examples of these are applications to optimal flow control problems (see [12], [13], 14]) and turbulence (see [8], [9], [15], [16]). In [17], though the tropical Pacific Ocean reduced gravity model was dealt with by the POD method, an exact theoretical analysis was not carried out. In particular, to the best of our knowledge, there are no published results addressing accuracy of the approximate solution of FDS derived with the POD and SVD for the tropical Pacific Ocean reduced gravity model.

In this paper, the POD and SVD techniques are used to study the FDS solutions for the tropical Pacific Ocean reduced gravity model. Ensembles of data are compiled from transient solutions computed from the equation system derived using FDS for the tropical Pacific Ocean reduced gravity model. Optimal orthogonal bases are used to reconstruct the elements of the ensemble with POD and SVD. Combining above results with a Galerkin projection procedure yields a new optimizing FDS model of lower dimensional order and of high accuracy for the tropical Pacific Ocean reduced gravity model. We show using numerical examples that the error between the optimizing POD approximate solution and the full FDS solution is consistent with theoretical error results, thus validating both the feasibility and efficiency of the POD method. Our method is different from the approaches used in Ref. [18] and [19], whose methods consist of Galerkin projection approaches where original variables are substituted for linear combination of POD bases and the error estimates therein are also derived. While the SVD approach combined with POD technology is used to treat the Burgers equation in [20] and the cavity flow problem in [21], the error estimates have not completely been derived, especially, an optimizing FDS has not been derived. Therefore, our method improves upon existing methods.

The paper is organized as follows. Section 2 is devoted to deriving the FDS based on staggered mesh for the tropical Pacific Ocean reduced gravity model and choosing the snapshots from transient solutions computed from the equation system derived by FDS. The optimal orthogonal bases are reconstructed from the elements of the snapshots with POD and SVD in Section 3. In Section 4, a new optimizing FDS model with lower dimensional number and high accuracy for the tropical Pacific Ocean reduced gravity model is obtained by combining the optimal orthogonal bases with a Galerkin projection procedure, an error estimate of the new optimizing FDS model is derived, and numerical examples are presented illustrating that the error between optimizing POD approximate solution and the full FDS solution is consistent with previously obtained theoretical results, thus validating the feasibility and efficiency of POD method. Section 5 provides main conclusions and gives future tentative idea. Finally, some of the error analysis are 
presented in Appendix A for the sake of the paper being self-contained.

\section{FDS FOR THE TROPICAL PACIFIC OCEAN REDUCED GRAVITY MODEL AND GENERATE SNAPSHOTS}

The variability of fluid flow and fluid total layer thickness over tropical oceans is an important question in studies of climate change and air-sea interaction. However, the accurate assessment of fluid flow and fluid total layer thickness is greatly limited due to the lack of direct measurements and an insufficient knowledge of air-sea exchange processes. The tropical Pacific Ocean reduced gravity model is a useful model for simulating fluid flow and fluid total layer thickness over tropical Pacific Ocean and it has been extensively applied to studying the ocean dynamics in tropical regions (see, [22] and [23]). The model consists of two layers above the thermocline with the same constant density. The ocean below the thermocline, with a higher density, is assumed to be sufficiently deep so that its velocity vanishes (Figure 1). The upper of the two active layers is a fixed-depth surface layer in which the thermodynamics are included. The surface layer communicates with the lower active layer through entrainment/ detrainment at their interface and through frictional horizontal shearing. We assume that there is no density difference across the base of the surface layer; that is, the surface layer is treated as part of the upper layer.

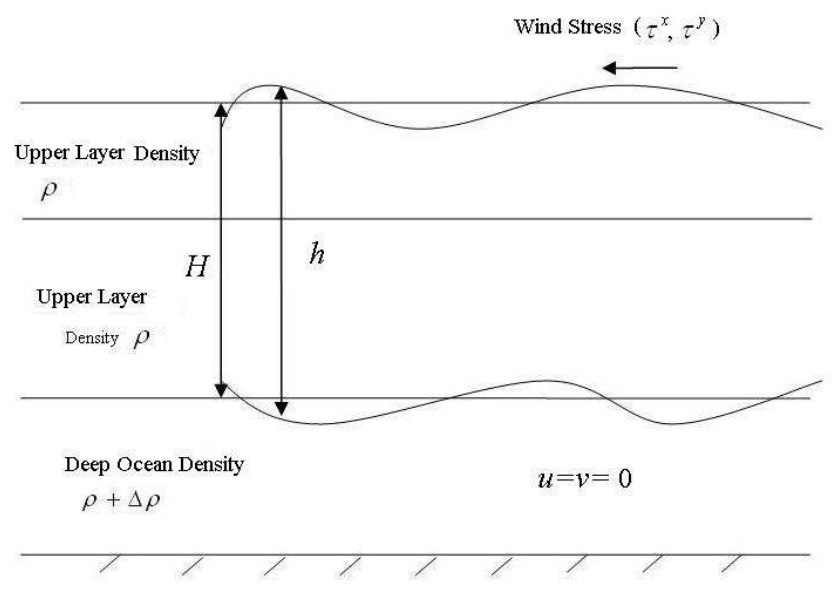

Figure 1. Ocean depth

Following Seager et al. (see [24]), the equations for the depth-averaged currents are written as:

Problem (I). Find $(u, v)$ and $h$ such that

$$
\begin{cases}\frac{\partial u}{\partial t}-f v=-g^{\prime} \frac{\partial h}{\partial x}+\frac{\tau^{x}}{\rho_{0} H}+A\left(\frac{\partial^{2} u}{\partial x^{2}}+\frac{\partial^{2} u}{\partial y^{2}}\right), & (x, y, t) \in \Omega \times(0, T), \\ \frac{\partial v}{\partial t}+f u=-g^{\prime} \frac{\partial h}{\partial y}+\frac{\tau^{y}}{\rho_{0} H}+A\left(\frac{\partial^{2} v}{\partial x^{2}}+\frac{\partial^{2} v}{\partial y^{2}}\right), & (x, y, t) \in \Omega \times(0, T), \\ \frac{\partial h}{\partial t}+H\left(\frac{\partial u}{\partial x}+\frac{\partial v}{\partial y}\right)=0, & (x, y, t) \in \Omega \times(0, T),\end{cases}
$$


where $(u, v)$ is the horizontal velocity of the depth-averaged currents; $h$ the total layer thickness; $f$ the Coriolis force; $H$ the mean depth of the layer (constant); $\rho_{0}$ the density of water; $g^{\prime}$ reduced gravity; and $A$ the horizontal eddy viscosity coefficient, $\left(\tau^{x}, \tau^{y}\right)$ the wind stress which is calculated by the aerodynamic bulk formula

$$
\left(\tau^{x}, \tau^{y}\right)=\rho_{a} C_{D} \sqrt{U_{\text {wind }}^{2}+V_{\text {wind }}^{2}}\left(U_{\text {wind }}, V_{\text {wind }}\right),
$$

here $\rho_{\alpha}$ is the density of the air; $C_{D}$ the wind stress drag coefficient; $\left(U_{\text {wind }}, V_{\text {wind }}\right)$ the wind velocity vector. The seasonal net surface heat flux over tropical oceans has been only simulated with the equations (1) to add to a thermodynamics equation by $\mathrm{Yu}$ and O'Brien (see, [25]). However, since the computational field over the tropical Pacific ocean is very extensive, and finite difference meshes are very dense and difficult to compute, fluid flow and fluid total layer thickness over tropical oceans are not simulated.

Let $\triangle x$ and $\triangle y$ be the spatial mesh size in $x$-direction and $y$-direction, respectively, and $\triangle t$ be the time step size $, u_{j+\frac{1}{2}, k}^{n}, v_{j, k+\frac{1}{2}}^{n}$, and $h_{j, k}^{n}$ denote function values of $u, v$, and $h$ at point $\left(x_{j+\frac{1}{2}}, y_{k}, t_{n}\right),\left(x_{j}, y_{k+\frac{1}{2}}, t_{n}\right)$, and $\left(x_{j}, y_{k}, t_{n}\right)(0 \leq j \leq J, 0 \leq k \leq K, 0 \leq$ $n \leq N=T / \triangle t)$, respectively.

In the following, we apply a staggered mesh (see Figure 2) FDS for solving Problem (I).

(1) Discretizing the continuous equation $\frac{\partial h}{\partial t}+H\left(\frac{\partial u}{\partial x}+\frac{\partial v}{\partial y}\right)=0$ yields

$$
\begin{aligned}
& h_{j, k}^{n+1}=h_{j, k}^{n}+H \triangle t\left(\frac{u_{j+\frac{1}{2}, k}-u_{j-\frac{1}{2}, k}}{\Delta x}+\frac{v_{j, k+\frac{1}{2}}-v_{j, k-\frac{1}{2}}}{\Delta y}\right)^{n+1} .
\end{aligned}
$$

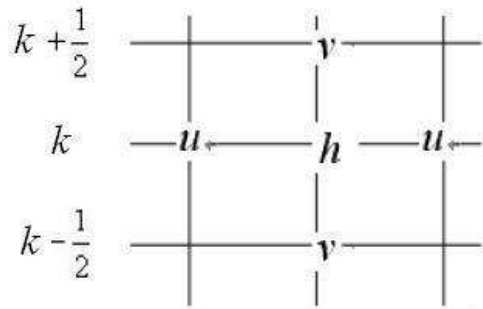

$$
\begin{aligned}
& j-\frac{1}{2} \quad j \quad j+\frac{1}{2}
\end{aligned}
$$

Figure 2. Staggered mesh graphics

(2) Discretizing the momentum equation

$$
\frac{\partial u}{\partial t}-f v=-g^{\prime} \frac{\partial h}{\partial x}+\frac{\tau^{x}}{\rho_{0} H}+A\left(\frac{\partial^{2} u}{\partial x^{2}}+\frac{\partial^{2} u}{\partial y^{2}}\right)
$$

in the $x$-direction at point $\left(x_{j+\frac{1}{2}}, y_{k}\right)$ yields

$$
u_{j+\frac{1}{2}, k}^{n+1}=F_{j+\frac{1}{2}, k}^{n}-g^{\prime} \frac{\Delta t}{\Delta x}\left[h_{j+1, k}^{n}-h_{j, k}^{n}\right]
$$


where

$$
\begin{aligned}
F_{j+\frac{1}{2}, k}^{n}= & u_{j+\frac{1}{2}, k}^{n}+\Delta t\left[f v+\frac{\tau^{x}}{\rho_{0} H}\right]_{j+\frac{1}{2}, k}^{n}+ \\
& A \Delta t\left[\frac{u_{j+\frac{1}{2}, k-1}-2 u_{j+\frac{1}{2}, k}+u_{j+\frac{1}{2}, k+1}}{\Delta y^{2}}+\frac{u_{j-\frac{1}{2}, k}-2 u_{j+\frac{1}{2}, k}+u_{j+\frac{3}{2}, k}}{\Delta x^{2}}\right]^{n},
\end{aligned}
$$

(3) Expanding the momentum equation

$$
\frac{\partial v}{\partial t}+f u=-g^{\prime} \frac{\partial h}{\partial y}+\frac{\tau^{y}}{\rho_{0} H}+A\left(\frac{\partial^{2} v}{\partial x^{2}}+\frac{\partial^{2} v}{\partial y^{2}}\right)
$$

in the $y$-direction at point $\left(x_{j}, y_{k+\frac{1}{2}}\right)$ yields

$$
v_{j, k+\frac{1}{2}}^{n+1}=G_{j, k+\frac{1}{2}}^{n}-g^{\prime} \frac{\Delta t}{\Delta y}\left[h_{j, k+1}^{n}-h_{j, k}^{n}\right]
$$

where

$$
\begin{aligned}
G_{j, k+\frac{1}{2}}^{n}= & v_{j, k}^{n}+\Delta t\left[\frac{\tau^{y}}{\rho_{0} H}-f u\right]_{j, k+\frac{1}{2}}^{n}+ \\
& A \Delta t\left[\frac{v_{j-1, k+\frac{1}{2}}-2 v_{j, k+\frac{1}{2}}+u_{j+1, k+\frac{1}{2}}}{\Delta x^{2}}+\frac{v_{j, k-\frac{1}{2}}-2 v_{j, k+\frac{1}{2}}+v_{j, k+\frac{3}{2}}}{\Delta y^{2}}\right]^{n} .
\end{aligned}
$$

Inserting (5) and (8) into (3) could obtain

$$
h_{j, k}^{n+1}=h_{j, k}^{n}+H \triangle t\left[\frac{h_{j-1, k}-2 h_{j, k}+h_{j+1, k}}{\Delta x^{2}}+\frac{h_{j, k-1}-2 h_{j, k}+h_{j, k+1}}{\Delta y^{2}}\right]^{n}+R,
$$

where

$$
R=\frac{1}{\Delta x}\left[F_{j+\frac{1}{2}, k}-F_{j-\frac{1}{2}, k}\right]^{n}+\frac{1}{\Delta y}\left[G_{j, k+\frac{1}{2}}-G_{j, k-\frac{1}{2}}\right]^{n} .
$$

Thus, if only the parameters $f, H, \rho_{0}, \rho_{a}, g^{\prime}, A, C_{D}$, the wind velocity vector $\left(U_{\text {wind }}, V_{\text {wind }}\right)$, time step size, and spatial step size in $x$ and $y$-directions are given, by solving (5), (8), and (10), one could obtain $u_{j+\frac{1}{2}, k}^{n}, v_{j, k+\frac{1}{2}}^{n}, h_{j, k}^{n}(0 \leq j \leq J, 0 \leq k \leq$ $K, 1 \leq n \leq N)$.

Write $u_{i}^{n}=u_{j+\frac{1}{2}, k}^{n}, v_{i}^{n}=v_{j, k+\frac{1}{2}}^{n}$, and $h_{i}^{n}=h_{j, k}^{n}, i=k J+j+1, m=J K(1 \leq i \leq$ $m, 0 \leq j \leq J-1,0 \leq k \leq K-1,0 \leq n \leq N)$. The $L \times m$ group of values consisting of the ensemble $\left\{u_{i}^{l}, v_{i}^{l}, h_{i}^{l}\right\}_{l=1}^{L}(1 \leq i \leq m)$ (usually $\left.L \ll N\right)$, known as "snapshots" which is useful and of interest for us, are chosen from $N \times m$ group of $\left\{u_{i}^{n}, v_{i}^{n}, h_{i}^{n}\right\}_{n=1}^{N}$ $(1 \leq i \leq m)$.

Remark 1. When one computes actual problems, one may obtain the ensemble of snapshots from physical system trajectories by drawing samples from experiments and interpolation (or data assimilation). For example, for weather forecast, one can use previous weather results to construct the ensemble of snapshots, then restructure the 
optimal basis for the ensemble of snapshots by following with POD, and finally combine it with a Galerkin projection to derive a reduced order dynamical system. Thus, the forecast of future weather change can be quickly simulated, which is of major importance for actual real-life applications.

\section{SINGULAR VALUE DECOMPOSITION AND POD OPTIMAL BASIS}

The POD method has received much attention in recent years as a tool to analyze complex physical systems. In this section, we use POD technique to deal with the "snapshots" obtained in previous Section 1 and to derive an "optimal" representation in an "average" sense.

The ensemble of snapshots $\left\{u_{i}^{l}, v_{i}^{l}, h_{i}^{l}\right\}_{l=1}^{L}(1 \leq i \leq m)$ can be expressed as three $m \times L$ matrices as follows:

$$
\left(\begin{array}{cccc}
u_{1}^{1} & u_{1}^{2} & \cdots & u_{1}^{L} \\
u_{2}^{1} & u_{2}^{2} & \cdots & u_{2}^{L} \\
\vdots & \vdots & \vdots & \vdots \\
u_{m}^{1} & u_{m}^{2} & \cdots & u_{m}^{L}
\end{array}\right),\left(\begin{array}{cccc}
v_{1}^{1} & v_{1}^{2} & \cdots & v_{1}^{L} \\
v_{2}^{1} & v_{2}^{2} & \cdots & v_{2}^{L} \\
\vdots & \vdots & \vdots & \vdots \\
v_{m}^{1} & v_{m}^{2} & \cdots & v_{m}^{L}
\end{array}\right), \quad\left(\begin{array}{cccc}
h_{1}^{1} & h_{1}^{2} & \cdots & h_{1}^{L} \\
h_{2}^{1} & h_{2}^{2} & \cdots & h_{2}^{L} \\
\vdots & \vdots & \vdots & \vdots \\
h_{m}^{1} & h_{m}^{2} & \cdots & h_{m}^{L}
\end{array}\right)
$$

If the means of columns of these matrices are denoted by

$$
\bar{u}^{l}=\frac{1}{m} \sum_{i=1}^{m} u_{i}^{l}, \quad \bar{v}^{l}=\frac{1}{m} \sum_{i=1}^{m} v_{i}^{l}, \quad \bar{h}^{l}=\frac{1}{m} \sum_{i=1}^{m} h_{i}^{l},
$$

then we can form new ensembles by focusing on deviations from the mean value as follows

$$
\left\{\tilde{u}_{i}^{l}\right\}_{l=1}^{L}, \quad\left\{\tilde{v}_{i}^{l}\right\}_{l=1}^{L}, \quad\left\{\tilde{h}_{i}^{l}\right\}_{l=1}^{L}, \quad 1 \leq i \leq m,
$$

where $\tilde{u}_{i}^{l}=u_{i}^{l}-\bar{u}^{l}, \tilde{v}_{i}^{l}=v_{i}^{l}-\bar{v}^{l}, \tilde{h}_{i}^{l}=h_{i}^{l}-\bar{h}^{l}(1 \leq i \leq m)$. Thus, we can reform new matrices as follows:

$$
A_{u}=\left(\begin{array}{cccc}
\tilde{u}_{1}^{1} & \tilde{u}_{1}^{2} & \cdots & \tilde{u}_{1}^{L} \\
\tilde{u}_{2}^{1} & \tilde{u}_{2}^{2} & \cdots & \tilde{u}_{2}^{L} \\
\vdots & \vdots & \vdots & \vdots \\
\tilde{u}_{m}^{1} & \tilde{u}_{m}^{2} & \cdots & \tilde{u}_{m}^{L}
\end{array}\right), A_{v}=\left(\begin{array}{cccc}
\tilde{v}_{1}^{1} & \tilde{v}_{1}^{2} & \cdots & \tilde{v}_{1}^{L} \\
\tilde{v}_{2}^{1} & \tilde{v}_{2}^{2} & \cdots & \tilde{v}_{2}^{L} \\
\vdots & \vdots & \vdots & \vdots \\
\tilde{v}_{m}^{1} & \tilde{v}_{m}^{2} & \cdots & \tilde{v}_{m}^{L}
\end{array}\right), A_{h}=\left(\begin{array}{cccc}
\tilde{h}_{1}^{1} & \tilde{h}_{1}^{2} & \cdots & \tilde{h}_{1}^{L} \\
\tilde{h}_{2}^{1} & \tilde{h}_{2}^{2} & \cdots & \tilde{h}_{2}^{L} \\
\vdots & \vdots & \vdots & \vdots \\
\tilde{h}_{m}^{1} & \tilde{h}_{m}^{2} & \cdots & \tilde{h}_{m}^{L}
\end{array}\right) .
$$

Define matrix norm $\|\cdot\|_{\alpha, \beta}$ as $\|A\|_{\alpha, \beta}=\sup _{\vec{x} \neq 0} \frac{\|A \vec{x}\|_{\alpha}}{\|\vec{x}\|_{\beta}} \quad$ (where $\|\cdot\|_{\alpha}$ and $\|\cdot\|_{\beta}$ are the norm of vector), the set $\Lambda$ as the collection of all normalized bases for $R^{m}$, and $\left\{\vec{\phi}_{1}, \vec{\phi}_{2}, \cdots, \vec{\phi}_{m}\right\} \subset \Lambda$ as an orthogonal basis for $R^{m}$. Then, finding "optimal" representation for $A_{u}, A_{v}$, and $A_{h}$ is formulated as the following optimization problem.

Optimization Problem (II). Find $\left\{\vec{\phi}_{1}, \vec{\phi}_{2}, \cdots, \vec{\phi}_{m}\right\} \subset \Lambda$, i.e., $\Phi=\left(\vec{\phi}_{1}, \vec{\phi}_{2}, \cdots\right.$, $\left.\vec{\phi}_{m}\right) \in R^{m \times m}$ such that it minimizes

$$
J(\Phi)=\left\|A_{u}-\Phi \Phi^{T} A_{u}\right\|_{2,2} .
$$


Thus, each column in the matrix $\Phi^{T} A_{u}$ is the set of coefficients used to expand the corresponding solutions in the new basis. And the POD basis is commonly defined by an orthonormal basis $\left\{\vec{\phi}_{1}, \vec{\phi}_{2}, \cdots, \vec{\phi}_{L}\right\}$ solving (16) for any $L \in\{1,2, \cdots, m\}$.

In order to obtain an "optimal" representation for $A_{u}$, we employ the Singular Value Decomposition (SVD), which is an important tool for finding optimal basis of optimization problem, to solve Optimization Problem (II). For matrix $A_{u} \in A^{m \times L}\left(A_{v}\right.$ and $A_{h}$ are similar), there exists the SVD

$$
A_{u}=U_{u}\left(\begin{array}{cc}
S_{u} & 0 \\
0 & 0
\end{array}\right) V_{u}^{T},
$$

where $U_{u} \in R^{m \times m}$ and $V_{u} \in R^{L \times L}$ are all orthogonal matrices and $S_{u}=\operatorname{diag}\left\{\sigma_{u 1}, \sigma_{u 2}\right.$, $\left.\cdots, \sigma_{u \ell}\right\} \in R^{\ell \times \ell}$ is the diagonal matrix correspondent to $A_{u} . \sigma_{u i}(i=1,2, \cdots, \ell)$ are the positive singular values. And the matrices $U_{u}=\left(\vec{\phi}_{u 1}, \vec{\phi}_{u 2}, \cdots, \vec{\phi}_{u m}\right) \in R^{m \times m}$ and $V_{u}=\left(\vec{\varphi}_{u 1}, \vec{\varphi}_{u 2}, \cdots, \vec{\varphi}_{u L}\right) \in R^{L \times L}$ contain the orthogonal eigenvectors to $A_{u} A_{u}^{T}$ and $A_{u}^{T} A_{u}$, respectively. The columns of these eigenvector matrices are organized so as to correspond to the singular values $\sigma_{u i}$ comprised in $S_{u}$ in a non-increasing order. And the singular values of the decomposition are connected to the eigenvalues of the matrices $A_{u} A_{u}^{T}$ and $A_{u}^{T} A_{u}$ in a manner such that $\lambda_{u i}=\sigma_{u i}^{2}(i=1,2, \cdots, \ell)$. Since the number of mesh points is by far larger than that of transient moment points, i.e., $m \gg L$, that is also that the order $m$ for matrix $A_{u} A_{u}^{T}$ is far larger than the order $L$ for matrix $A_{u}^{T} A_{u}$, however their null eigenvalues are identical, therefore, we may first solve the eigen equation corresponding to matrix $A_{u}^{T} A_{u}$ to find the eigenvectors $\vec{\varphi}_{u j}(j=1,2, \cdots, L)$, and then by relationship

$$
\vec{\phi}_{u j}=A_{u} \vec{\varphi}_{u j} / \sigma_{u j}, \quad j=1,2, \cdots, \ell,
$$

we may obtain $\ell(\ell \leq L)$ eigenvectors corresponding to the non-null eigenvalues for matrix $A_{u} A_{u}^{T}$, where formula (18) just follows from SVD.

Using the relationship properties of spectral radius and $\|\cdot\|_{2,2}$ for matrices, if $M_{u}<$ $r=\operatorname{rank} A_{u}(r \leq \ell \leq L)$, then we obtain the following equation

$$
\sigma_{u\left(M_{u}+1\right)}=\min _{\operatorname{rank}(B) \leq M_{u}}\left\|A_{u}-B\right\|_{2,2}=\left\|A_{u}-A_{M_{u}}\right\|_{2,2},
$$

where $A_{M_{u}}=\sum_{i=1}^{M_{u}} \sigma_{u i} \vec{\phi}_{u i} \vec{\varphi}_{u i}^{T}, \vec{\phi}_{u i}\left(i=1,2, \cdots, M_{u}\right)$ and $\vec{\varphi}_{u j}\left(j=1,2, \cdots, M_{u}\right)$ are $M_{u}$ first column vectors of matrices $U_{u}$ and $V_{u}$, respectively.

By comparing the Optimization Problem (II) with Eq.(19), it is obvious that the minimum distance between the matrix $A_{u}$ and $B$ (which is of rank $M_{u}$ ), or the optimal approximation of $A_{u}$ by $B$ is obtained by using the matrix $A_{M_{u}}$ defined in Eq.(19), i.e., the $A_{M_{u}}$ should be the optimal representation of $A_{u}$ in the optimal base. Thus, the optimal base is found in the construction of $A_{M_{u}}$. By using the property of eigenvector, 
it is well known that $\Phi_{u}=U_{u}=\left(\vec{\phi}_{u 1}, \vec{\phi}_{u 2}, \cdots, \vec{\phi}_{M_{u}}\right)\left(M_{u} \ll L\right)$ is a group of the optimal base for Optimization Problem (II).

Denote the $L$ column vectors of matrices $A_{u}$ by $\vec{a}_{u}^{l}=\left(\tilde{u}_{1}^{l}, \tilde{u}_{2}^{l}, \cdots, \tilde{u}_{m}^{l}\right)^{T}(l=1,2, \cdots$, $L)$, and $\varepsilon_{l}(l=1,2, \cdots, L)$ by unit column vectors except that a vector component is 1 , while the other components are 0 . Then by the compatibility of the norm for matrices and vectors, we obtain

$$
\left\|\vec{a}_{u}^{l}-P_{M_{u}}\left(\vec{a}_{u}^{l}\right)\right\|_{2}=\left\|\left(A_{u}-A_{M_{u}}\right) \varepsilon_{n}\right\|_{2} \leq\left\|A_{u}-A_{M_{u}}\right\|_{2,2}\left\|\varepsilon_{n}\right\|_{2}=\sqrt{\lambda_{u\left(M_{u}+1\right)}},
$$

where $P_{M_{u}}\left(\vec{a}_{u}^{l}\right)=\sum_{j=1}^{M_{u}}\left(\vec{\phi}_{u j}, \vec{a}_{u}^{j}\right) \vec{\phi}_{u j},\left(\vec{\phi}_{u j}, \vec{a}_{u}^{j}\right)$ is the canonical inner product for vector $\vec{\phi}_{u j}$ and vector $\vec{a}_{u}^{j}$. Inequality (20) shows that $P_{M_{u}}\left(\vec{a}_{u}^{l}\right)$ is the optimal approximation to $\vec{a}_{u}^{l}$, whose error is $\sqrt{\lambda_{u\left(M_{u}+1\right)}}$.

By the same approach as the above $(20)$, if $\vec{a}_{v}^{l}=\left(\tilde{v}_{1}^{l}, \tilde{v}_{2}^{l}, \cdots, \tilde{v}_{m}^{l}\right)^{T}$ and $\vec{a}_{h}^{l}=\left(\tilde{h}_{1}^{l}, \tilde{h}_{2}^{l}, \cdots\right.$, $\left.\tilde{h}_{m}^{l}\right)^{T}(l=1,2, \cdots, L)$ are $L$ column vectors of matrices $A_{v}$ and $A_{h}$, respectively, then $P_{M_{v}}\left(\vec{a}_{v}^{l}\right)=\sum_{j=1}^{M_{v}}\left(\vec{\phi}_{v j}, \vec{a}_{v}^{j}\right) \vec{\phi}_{v j}$ and $P_{M_{h}}\left(\vec{a}_{h}^{l}\right)=\sum_{j=1}^{M_{h}}\left(\vec{\phi}_{h j}, \vec{a}_{h}^{j}\right) \vec{\phi}_{h j}$ are respectively the optimal approximations to $\vec{a}_{v}^{l}$ and $\vec{a}_{h}^{l}$, whose errors are respectively $\sqrt{\lambda_{v\left(M_{v}+1\right)}}$ and $\sqrt{\lambda_{h\left(M_{h}+1\right)}}$, i.e.,

$$
\begin{gathered}
\left\|\vec{a}_{v}^{l}-P_{M_{v}}\left(\vec{a}_{v}^{l}\right)\right\|_{2} \leq \sqrt{\lambda_{v\left(M_{v}+1\right)}}, \\
\left\|\vec{a}_{h}^{l}-P_{M_{h}}\left(\vec{a}_{h}^{l}\right)\right\|_{2} \leq \sqrt{\lambda_{h\left(M_{h}+1\right)}},
\end{gathered}
$$

where $\lambda_{v\left(M_{v}+1\right)}$ is $\left(M_{v}+1\right)$-th eigenvalue for $A_{v} A_{v}^{T}$ and $\lambda_{h\left(M_{h}+1\right)}$ is $\left(M_{h}+1\right)$-th eigenvalue for $A_{h}^{T} A_{h}$, and $\Phi_{v}=U_{v}=\left(\vec{\phi}_{v 1}, \vec{\phi}_{v 2}, \cdots, \vec{\phi}_{v M_{v}}\right)$ and $\Phi_{h}=U_{h}=\left(\vec{\phi}_{h 1}, \vec{\phi}_{h 2}, \cdots, \vec{\phi}_{h M_{h}}\right)$ are the optimal bases corresponding to $A_{v}$ and $A_{h}$, respectively.

\section{OPTIMIZING NUMERICAL SOLUTION AND SIMULATION FOR THE TROPICAL PACIFIC OCEAN REDUCED GRAVITY MODEL}

\subsection{Optimizing numerical solution for the tropical Pacific Ocean reduced gravity model}

In this section, we construct the optimizing solution for the tropical Pacific Ocean reduced gravity model using optimal bases $\Phi_{u}=\left(\vec{\phi}_{u 1}, \vec{\phi}_{u 2}, \cdots, \vec{\phi}_{M_{u}}\right), \Phi_{v}=\left(\vec{\phi}_{v 1}, \vec{\phi}_{v 2}, \cdots, \vec{\phi}_{M_{v}}\right)$, and $\Phi_{h}=\left(\vec{\phi}_{h 1}, \vec{\phi}_{h 2}, \cdots, \vec{\phi}_{M_{h}}\right)\left(M_{u}, M_{v}, M_{h} \ll L\right)$, and it is shown by considering results obtained for the numerical simulation of tropical Pacific Ocean reduced gravity model that this validates the feasibility and efficiency of POD method.

Write

$$
\left\{\begin{array}{c}
\vec{u}_{m}(t)=\left(u_{1}(t), u_{2}(t), \cdots, u_{m}(t)\right)^{T} \\
\vec{v}_{m}(t)=\left(v_{1}(t), v_{2}(t), \cdots, v_{m}(t)\right)^{T} \\
\vec{h}_{m}(t)=\left(h_{1}(t), h_{2}(t), \cdots, h_{m}(t)\right)^{T}
\end{array}\right.
$$


where $u_{i}=u_{j+\frac{1}{2}, k}, v_{i}=v_{j, k+\frac{1}{2}}$, and $h_{i}=h_{j, k}(1 \leq i \leq m, i=k J+j+1, m=J K$, $0 \leq j \leq J-1,0 \leq k \leq K-1,0 \leq n \leq N)$. Thus, (5), (8), and (10) are written as the following vector formulation:

$$
\left(\vec{u}_{m}^{n+1}, \vec{v}_{m}^{n+1}, \vec{h}_{m}^{n+1}\right)^{T}=\left(\vec{u}_{m}^{n}, \vec{v}_{m}^{n}, \vec{h}_{m}^{n}\right)^{T}+\Delta t \tilde{F}\left(\vec{u}_{m}^{n}, \vec{v}_{m}^{n}, \vec{h}_{m}^{n}\right)
$$

where $\tilde{F}\left(\vec{u}_{m}^{n}, \vec{v}_{m}^{n}, \vec{h}_{m}^{n}\right)=\left(\tilde{F}_{1}\left(\vec{u}_{m}^{n}, \vec{v}_{m}^{n}, \vec{h}_{m}^{n}\right), \tilde{F}_{2}\left(\vec{u}_{m}^{n}, \vec{v}_{m}^{n}, \vec{h}_{m}^{n}\right), \tilde{F}_{3}\left(\vec{u}_{m}^{n}, \vec{v}_{m}^{n}, \vec{h}_{m}^{n}\right)\right)^{T}$ is the vector function following from (5), (6) and (8) (11). Put

$$
\left(\overrightarrow{\tilde{u}}_{m}^{n}, \overrightarrow{\tilde{v}}_{m}^{n}, \overrightarrow{\tilde{h}}_{m}^{n}\right)^{T}=\left(\Phi_{u} \vec{\alpha}_{M_{u}}^{n}, \Phi_{v} \vec{\beta}_{M_{v}}^{n}, \Phi_{h} \vec{\gamma}_{M_{h}}^{n}\right)^{T}
$$

where $\overrightarrow{\tilde{u}}_{m}^{n}=\left(\tilde{u}_{1}^{n}, \tilde{u}_{2}^{n}, \cdots, \tilde{u}_{m}^{n}\right)^{T}, \overrightarrow{\tilde{v}}_{m}^{n}=\left(\tilde{v}_{1}^{n}, \tilde{v}_{2}^{n}, \cdots, \tilde{v}_{m}^{n}\right)^{T}, \overrightarrow{\tilde{h}}_{m}^{n}=\left(\tilde{h}_{1}^{n}, \tilde{h}_{2}^{n}, \cdots, \tilde{h}_{m}^{n}\right)^{T}, \tilde{u}_{i}^{n}=$ $u_{i}^{n}-\bar{u}^{n}, \tilde{v}_{i}^{n}=v_{i}^{n}-\bar{v}^{n}, \tilde{h}_{i}^{n}=h_{i}^{n}-\bar{h}^{n}(1 \leq i \leq m)$, and $\bar{u}^{n}, \bar{v}^{n}$, and $\bar{h}^{n}$ are given in (13).

Inserting (25) into (24), and noting that $\Phi_{u}, \Phi_{v}$, and $\Phi_{h}$ are orthogonal matrices, we may obtain the reduced model which has $M_{u}+M_{v}+M_{h}\left(M_{u}, M_{v}, M_{h} \ll L \ll m\right)$ unknown values:

$$
\left(\vec{\alpha}_{M_{u}}^{n+1}, \vec{\beta}_{M_{v}}^{n+1}, \vec{\gamma}_{M_{h}}^{n+1}\right)^{T}=\left(\vec{\alpha}_{M_{u}}^{n}, \vec{\beta}_{M_{v}}^{n}, \vec{\gamma}_{M_{h}}^{n}\right)^{T}+\tilde{G}\left(\vec{\alpha}_{M_{u}}^{n}, \vec{\beta}_{M_{v}}^{n}, \vec{\gamma}_{M_{h}}^{n}\right), n=0,1,2, \cdots,
$$

where $\tilde{G}\left(\vec{\alpha}_{M_{u}}^{n}, \vec{\beta}_{M_{v}}^{n}, \vec{\gamma}_{M_{h}}^{n}\right)=\Delta t\left(\Phi_{u}^{T} \tilde{F}_{1}\left(\Phi_{u} \vec{\alpha}_{M_{u}}^{n}+\bar{u}^{n} I_{m}, \Phi_{v} \vec{\beta}_{M_{v}}^{n}+\bar{v}^{n} I_{m}, \Phi_{h} \vec{\gamma}_{M_{h}}^{n}+\bar{h}^{n} I_{m}\right)\right.$, $\Phi_{v}^{T} \tilde{F}_{2}\left(\Phi_{u} \vec{\alpha}_{M_{u}}^{n}+\bar{u}^{n} I_{m}, \Phi_{v} \vec{\beta}_{M_{v}}^{n}+\bar{v}^{n} I_{m}, \Phi_{h} \vec{\gamma}_{M_{h}}^{n}+\bar{h}^{n} I_{m}\right), \Phi_{h}^{T} \tilde{F}_{3}\left(\Phi_{u} \vec{\alpha}_{M_{u}}^{n}+\bar{u}^{n} I_{m}, \Phi_{v} \vec{\beta}_{M_{v}}^{n}+\right.$ $\left.\left.\bar{v}^{n} I_{m}, \Phi_{h} \vec{\gamma}_{M_{h}}^{n}+\bar{h}^{n} I_{m}\right)\right)^{T}+\left(\Phi_{u}^{T}\left(\bar{u}^{n}-\bar{u}^{n+1}\right) I_{m}, \Phi_{v}^{T}\left(\bar{v}^{n}-\bar{v}^{n+1}\right) I_{m}, \Phi_{h}^{T}\left(\bar{h}^{n}-\bar{h}^{n+1}\right) I_{m}\right)^{T}, I_{m}=$ $(1)_{m \times 1}$, which initial values are $\vec{\alpha}_{M_{u}}^{0}=\Phi_{u}^{T} \overrightarrow{\tilde{u}}_{m}^{0}, \vec{\beta}_{M_{v}}^{0}=\Phi_{v}^{T} \overrightarrow{\tilde{v}}_{m}^{0}, \vec{\gamma}_{M_{h}}^{0}=\Phi_{h}^{T} \overrightarrow{\tilde{h}}_{m}^{0}$.

After one has obtained $\vec{\alpha}_{M_{u}}^{n}, \vec{\beta}_{M_{v}}^{n}$, and $\vec{\gamma}_{M_{h}}^{n}$ from (26), one obtains the POD optimal solutions for Problem (I) by $u_{i}^{* n}=\tilde{u}_{i}^{n}+\bar{u}^{n}, v_{i}^{* n}=\tilde{v}_{i}^{n}+\bar{v}^{n}, h_{i}^{* n}=\tilde{h}_{i}^{n}+\bar{h}^{n}$ and (25), where $\bar{u}^{n}=\frac{1}{m} \sum_{i=1}^{m} u_{i}^{n}, \bar{v}^{n}=\frac{1}{m} \sum_{i=1}^{m} v_{i}^{n}, \bar{h}^{n}=\frac{1}{m} \sum_{i=1}^{m} h_{i}^{n}(1 \leq n \leq N)$. Thus, we get the optimal numerical solutions which are written as $\left(u_{j+\frac{1}{2}, k}^{* n}, v_{j, k+\frac{1}{2}}^{* n}, h_{j, k}^{* n}\right)(0 \leq j \leq J-1,0 \leq$ $k \leq K-1,0 \leq n \leq N)$ for Problem (I), where $u_{j+\frac{1}{2}, k}^{* n}=u_{i}^{* n}, v_{j, k+\frac{1}{2}}^{* n}=v_{i}^{* n}, h_{j, k}^{* n}=h_{i}^{* n}$ $(j=i-1-k J \geq 0,1 \leq i \leq m, 0 \leq k \leq K-1,0 \leq n \leq N)$.

Remark 2. If $0 \leq n=l \leq L$, the solution vectors obtained by $P_{M_{u}}\left(\vec{a}_{u}^{l}\right)=$ $\sum_{j=1}^{M_{u}}\left(\vec{\phi}_{u j}, \vec{a}_{u}^{j}\right) \vec{\phi}_{u j}, P_{M_{v}}\left(\vec{a}_{v}^{l}\right)=\sum_{j=1}^{M_{v}}\left(\vec{\phi}_{v j}, \vec{a}_{v}^{j}\right) \vec{\phi}_{v j}$, and $P_{M_{h}}\left(\vec{a}_{h}^{l}\right)=\sum_{j=1}^{M_{h}}\left(\vec{\phi}_{h j}, \vec{a}_{h}^{j}\right) \vec{\phi}_{h j}$ are the same as that the above optimal method (25) and (26). However, the above equations (25) and (26) can be used to compute the transient solutions backward the longest time (at this moment, one doesn't have to take deviations from the mean value, and can directly take $\bar{u}^{l}=\bar{v}^{l}=\bar{h}^{l}=0$ ), that is, one can use present data to forecast model future evolution. Therefore, POD-FDS is a method with extensive applications. Especially, (26) has only $\left(M_{u}+M_{v}+M_{h}\right) \times n$ unknown values, but (5), (8), and (10) have $3 m \times n(n=1,2,3, \cdots)$ unknown values (usually $M_{u}, M_{v}, M_{h} \ll L \ll m$ ). 
Remark 3. By (20)-(22), we have

$$
\left\{\begin{array}{l}
\left|u_{j+\frac{1}{2}, k}^{l}-u_{j+\frac{1}{2}, k}^{* l}\right| \leq\left\|\vec{a}_{u}-P_{M_{u}}\left(\vec{a}_{u}^{l}\right)\right\|_{2}=\sqrt{\lambda_{u\left(M_{u}+1\right)}}, \\
\left|v_{j, k+\frac{1}{2}}^{l}-v_{j, k+\frac{1}{2}}^{* l}\right| \leq\left\|\vec{a}_{v}-P_{M_{v}}\left(\vec{a}_{v}^{l}\right)\right\|_{2}=\sqrt{\lambda_{v\left(M_{v}+1\right)}}, \\
\left|h_{j, k}^{l}-h_{j, k}^{* l}\right| \leq\left\|\vec{a}_{h}-P_{M_{h}}\left(\vec{a}_{h}^{l}\right)\right\|_{2}=\sqrt{\lambda_{h\left(M_{h}+1\right)}}, 1 \leq l \leq L,
\end{array}\right.
$$

where $|\cdot|$ is the absolute value of real number. Since the error of numerical solutions $\left(u_{j+\frac{1}{2}, k}^{n}, v_{j, k+\frac{1}{2}}^{n}, h_{j, k}^{n}\right)(1 \leq n \leq N, 1 \leq j \leq J, 1 \leq k \leq K)$ for Problem (I) obtained by (5), (8), and (10) (see [1] or [2], or see Appendix A) is

$$
\begin{aligned}
\left|E_{n}\left(u_{j+\frac{1}{2}, k}^{n}, v_{j, k+\frac{1}{2}}^{n}, h_{j, k}^{n}\right)\right|= & \mid\left(u\left(x_{j+\frac{1}{2}}, y_{k}, t_{n}\right), v\left(x_{j}, y_{k+\frac{1}{2}}, t_{n}\right), h\left(x_{j}, y_{k}, t_{n}\right)\right) \\
& -\left(u_{j+\frac{1}{2}, k}^{n}, v_{j, k+\frac{1}{2}}^{n}, h_{j, k}^{n}\right) \mid=O\left(\Delta t, \Delta x^{2}, \Delta y^{2}\right)
\end{aligned}
$$

if the solution $(u(x, y, t), v(x, y, t), h(x, y, t))$ for Problem (I) has more regularity, the error of optimal numerical solutions obtaining from (24)-(26) is, by (20)-(22) and (28),

$$
\begin{aligned}
\left|E\left(u_{j+\frac{1}{2}, k}^{* l}, v_{j, k+\frac{1}{2}}^{* l}, h_{j, k}^{* l}\right)\right|= & \mid\left(u\left(x_{j+\frac{1}{2}}, y_{k}, t_{l}\right), v\left(x_{j}, y_{k+\frac{1}{2}}, t_{l}\right), h\left(x_{j}, y_{k}, t_{l}\right)\right) \\
& -\left(u_{j+\frac{1}{2}, k}^{* l}, v_{j, k+\frac{1}{2}}^{* l}, h_{j, k}^{* l}\right) \mid \\
\leq & \mid\left(u\left(x_{j+\frac{1}{2}}, y_{k}, t_{l}\right), v\left(x_{j}, y_{k+\frac{1}{2}}, t_{l}\right), h\left(x_{j}, y_{k}, t_{l}\right)\right) \\
& -\left(u_{j+\frac{1}{2}, k}^{l}, v_{j, k+\frac{1}{2}}^{l}, h_{j, k}^{l}\right) \mid \\
& +\left|\left(u_{j+\frac{1}{2}, k}^{l}, v_{j, k+\frac{1}{2}}^{l}, h_{j, k}^{l}\right)-\left(u_{j+\frac{1}{2}, k}^{* l}, v_{j, k+\frac{1}{2}}^{* l}, h_{j, k}^{* l}\right)\right| \\
= & O\left(\Delta t, \Delta x^{2}, \Delta y^{2}, \sqrt{\lambda_{u\left(M_{u}+1\right)}}, \sqrt{\lambda_{v\left(M_{v}+1\right)}}, \sqrt{\lambda_{h\left(M_{h}+1\right)}}\right),
\end{aligned}
$$

where $l=1,2, \cdots, L$.

\subsection{Numerical simulation for the tropical Pacific Ocean reduced gravity model}

In this study, we applied the model to the tropic Pacific Ocean domain $\left(29^{\circ} \mathrm{S} \sim 29^{\circ} \mathrm{N}\right.$, $\left.120^{\circ} \mathrm{E} \sim 70^{\circ} \mathrm{W}\right)$, using parameters values of $f=2(7.29 E-5) \sin (x, y), H=150 \mathrm{~m}, \rho_{0}=$ $1.2 \mathrm{~kg} \cdot \mathrm{m}^{-3}, \rho_{a}=1025 \mathrm{~kg} \cdot \mathrm{m}^{-3}, g^{\prime}=3.7 \times 10^{-2}, A=750 \mathrm{~m}^{2} \mathrm{sec}^{-1}$, and $C_{D}=1.5 \times 10^{-3}$ for Problem (I). This chosen model domain allows all possible equatorially trapped waves to be excited by the applied wind forcing (see [25]). We choose the spatial mesh size for the dynamical model to be $\Delta x=\Delta y=0.5^{\circ}$ and the time mesh size to be $\Delta t=100 \mathrm{~s}$. The model is driven by the Florida State University (FSU) climatology monthly mean winds ( cf. [26]), and the data are projected onto each time step by a linear interpolation and onto each grid point by a bilinear interpolation, for instance, in January (see Figure 3 
(a)) and in July (see Figure 3 (b)).

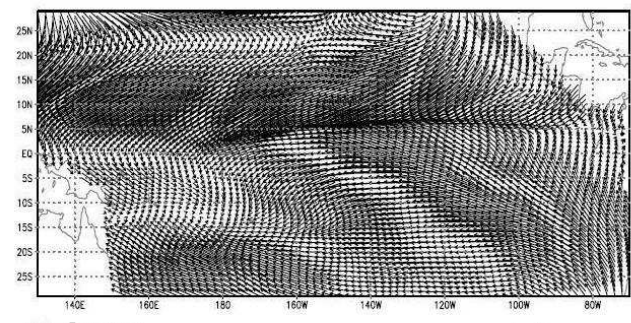

(a) Jannary

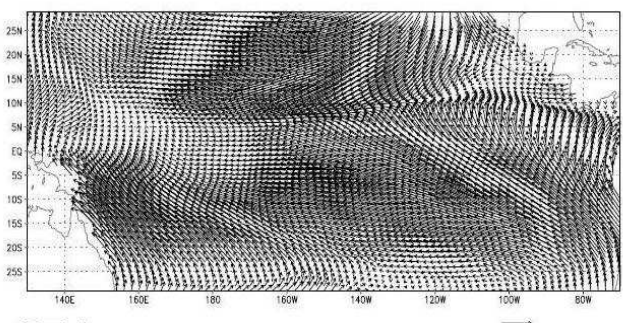

(b) July

Figure 3. Wind velocity vector for January and for July

We choose 20 or 30 values (i.e., snapshots) from the solutions solving (5), (8), and (10) for 1 year. It is shown by computing that eigenvalues $\lambda_{u 8}, \lambda_{v 8}$, and $\lambda_{h 8}$ are satisfying $\max \left\{\sqrt{\lambda_{u 8}}, \sqrt{\lambda_{v 8}}, \sqrt{\lambda_{h 8}}\right\} \leq 10^{-3}$. We obtain POD-FDS solution for fluid total layer thickness $h$ depicted graphically in Figure 4(a) and Figure 4(b), respectively, where the red curves represent numerical solution for 20 snapshots using 7 optimal bases to solve (24)-(26), the green curves represent numerical solution for 30 snapshots using 7 optimal bases to solve (24)-(26), and the black curves represent the solutions solving equation (5), (8), and (10) on January and on July.

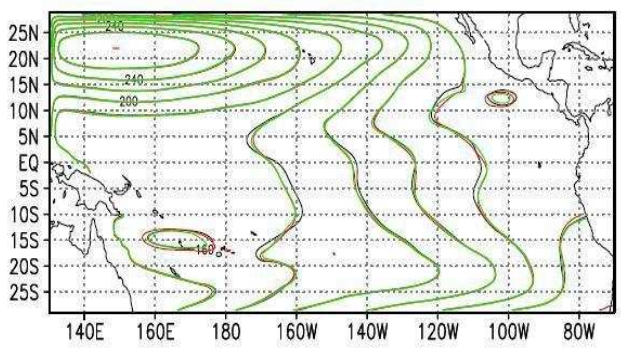

(a) Jannary

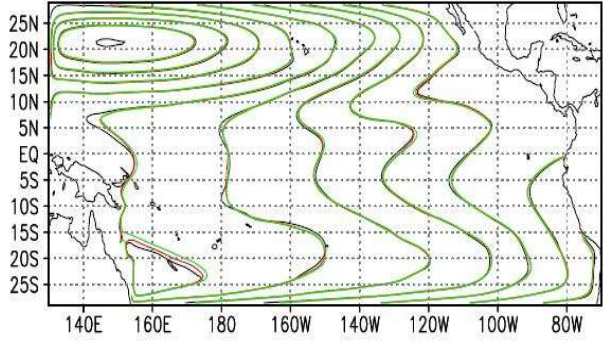

(b) July

Figure 4. Fluid total layer thickness for full bases (black curve), 7 optimal bases for 20 POD bases (red curve), and 7 optimal bases for 30 POD bases for month of January (Figure (a)) and July (Figure (b))

We also obtain POD-FDS solution for fluid velocity on longitude direction $u$ depicted graphically in Figure 5(c) and Figure 5(d),respectively, for 20 snapshots using 7 optimal bases to solve (24)-(26), and Figure 5(a) and Figure 5(b) are respectively the solutions 
solving equations (5), (8), and (10) for January and for July.
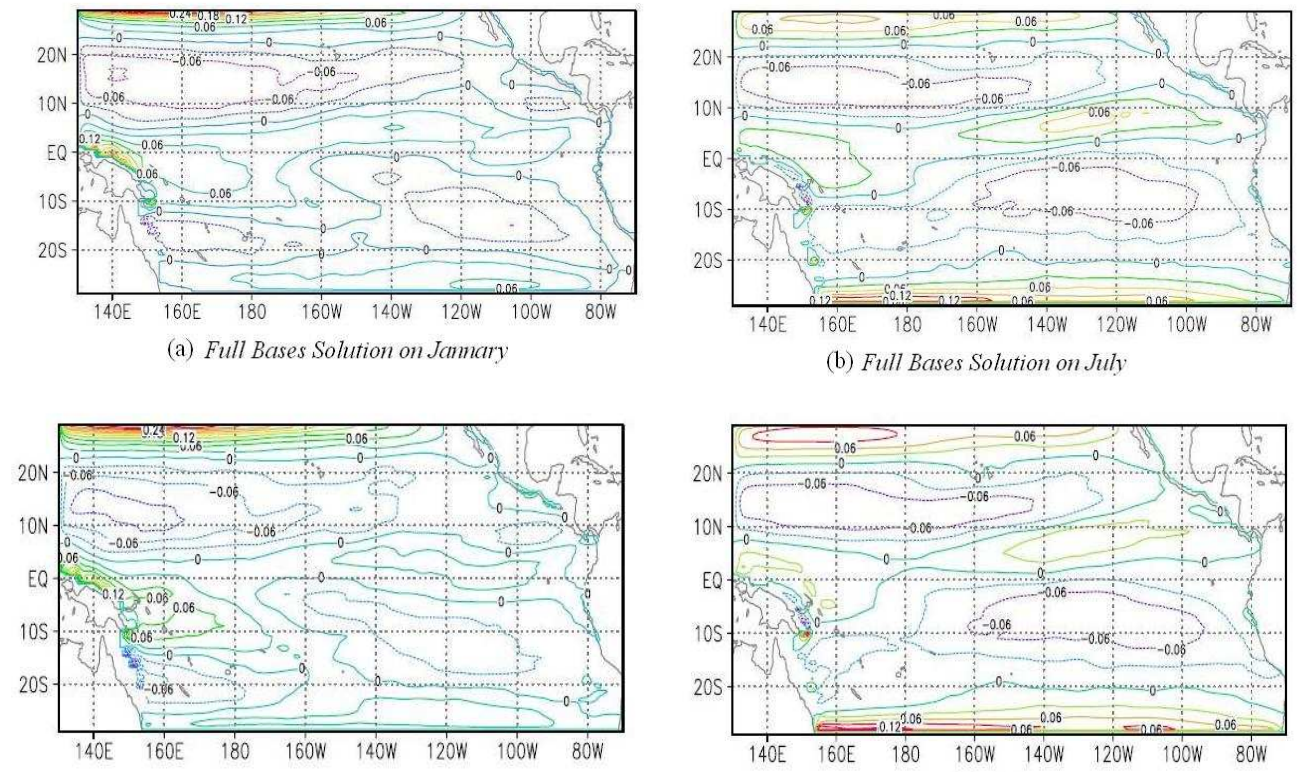

(c) POD Solution on Jannary

(d) POD Solution on Jannary

Figure 5. Fluid velocity in longitude direction for full bases and 20 POD bases

The POD-FDS solution for fluid velocity in the latitude direction $u$ for 20 snapshots using 7 optimal bases to solve (24)-(26) for January and for July are depicted graphically in Figure 6(c) and Figure 6(d), respectively, and the solutions solving equation (5), (8), and (10) are depicted graphically in Figure 6(a) and Figure 6(b), respectively.

Figure 7 shows the errors between numerical solutions obtained with a different number of optimal bases and solutions obtained with (5), (8), and (10), where the red curve represents the error of fluid total layer thickness, the blue curve represents the error of fluid velocity in the longitude direction, and the green curve represents the error of fluid velocity in the latitude direction in Figure 7. It is shown by comparing results for full and POD reduced model that the computational load for velocity vector and fluid total layer thickness with POD-FDS is sizably reduced, and the error between them does not exceed $2 \times 10^{-3}$. And the results of the error for the actual example are consistent with theoretical results obtained by computing with Eq.(29). This also shows that finding the approximate solutions for the tropical Pacific Ocean reduced gravity model with POD-FDS is computationally very effective. This also shows that finding the approximate solutions for the tropical Pacific Ocean reduced gravity model with POD-FDS is 
computationally very effective.

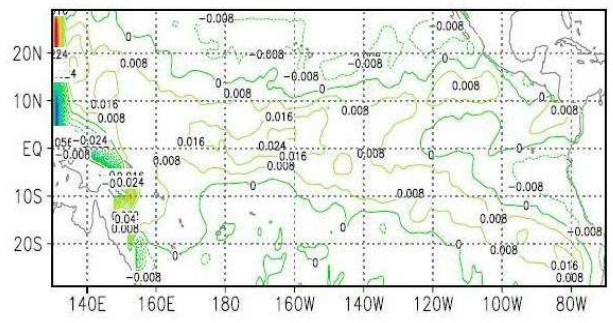

(a) Full Basse Solution on Jannary

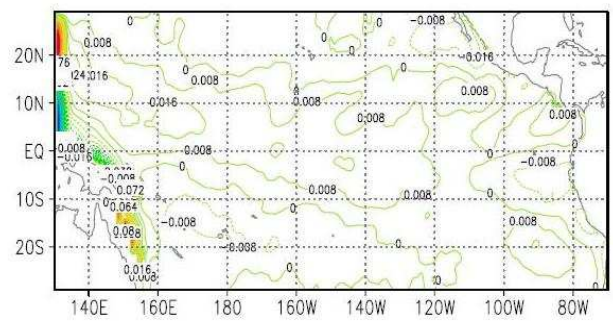

(c) POD Solution on Jannary

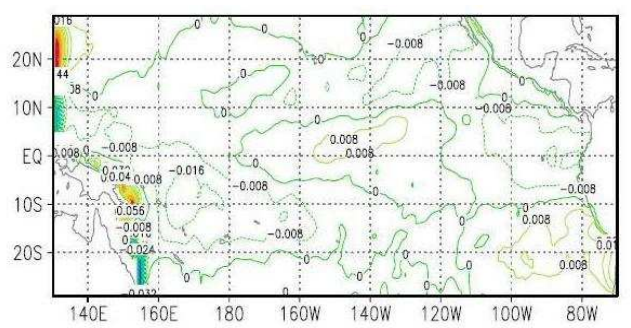

(b) Full bases Solution on July

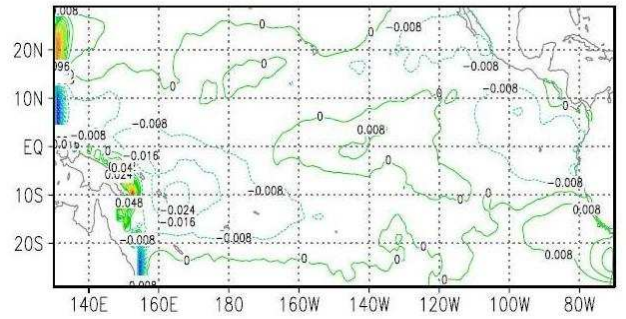

(d) POD Solution on July

Figure 6. Fluid velocity in latitude direction for full bases and 20 POD bases
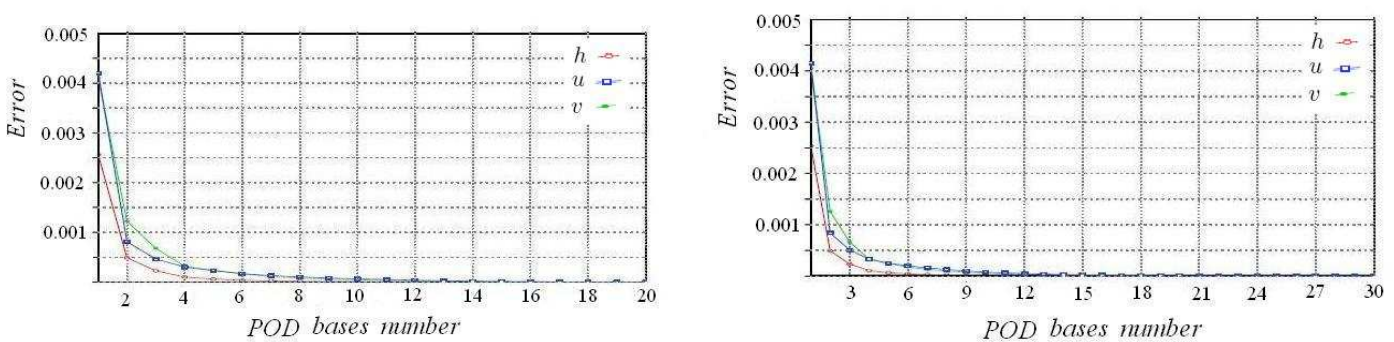

Figure 7. Error of numerical solutions of different optimal bases for 20 and 30 snapshots

Remark 4. Equations (24)-(26) to solve the tropical Pacific Ocean reduced gravity model only contain $21 n$ unknown values since $M_{u}=M_{v}=M_{h}=7$, in addition, order 20 eigenproblems which are easily solved using the Matlab software package, however, equations (5), (8), and (10) contain $3 \times 121 \times 321 n$ unknown values since the computing domain is $29^{\circ} \mathrm{S} \sim 29^{\circ} \mathrm{N}$ and $120^{\circ} \mathrm{E} \sim 70^{\circ} \mathrm{W}$, and the spatial mesh size for the dynamical model is chosen as $\Delta x=\Delta y=0.5^{\circ}$. Therefore, our optimizing reduced order FDS does alleviate the computational load and saves time-consuming calculations and resource demands in the actual computational process, in a way that guarantees a sufficiently accurate numerical solution as shown by the error analysis provided. The results of the error for the actual example are consistent with theoretical results obtained by computing with Eq.(29). This also shows that finding the approximate solutions for the tropical Pacific Ocean reduced gravity model with POD-FDS is computationally very effective. 


\section{CONCLUSIONS}

In this paper, we have employed the POD and SVD techniques to study the FDS solutions for the tropical Pacific Ocean reduced gravity model and to reconstruct optimal orthogonal bases of ensembles of data which are compiled from transient solutions derived by using FDS the equation system. We have also combined the optimal orthogonal bases with a Galerkin projection procedure, thus yielding a new optimizing FDS model of lower dimensional order and of high accuracy for the tropical Pacific Ocean reduced gravity model. We have then proceeded to derive error estimates between our optimizing FDS approximate solutions and the usual full order FDS numerical solutions, and have shown using numerical examples that the error between the optimizing POD approximate solution and the full FDS solution is consistent with the theoretical error results, thus validating both feasibility and efficiency of our optimizing FDS. Future work in this area will aim to extend the optimizing FDS, by implementing it to a realistic sea forecast system and to more complicated system of PDEs, for instance, the nonlinear shallow water equation system consisting of water dynamics equations, the silt transport equation and the equation of bottom topography change. We have shown both by theoretical analysis as well as by numerical examples that the optimizing FDS presented herein has extensive perspective applications.

\section{REFERENCES}

1. Chung T. Computational Fluid Dynamics. Cambridge University Press, Cambridge, UK, 2002, 1036pp.

2. Xin XK, Liu RX, Jiang BC. Computational Fluid Dynamics. National Defense Science Technology Press, Changsha, 1989 (in Chinese).

3. Holmes P, Lumley JL, Berkooz G. Turbulence, Coherent Structures, Dynamical Systems and Symmetry. Cambridge University Press, Cambridge, UK, 1996.

4. Fukunaga K. Introduction to Statistical Recognition. Academic Press, 1990.

5. Jolliffe IT, Principal Component Analysis. Springer-Verlag, 2002.

6. Crommelin DT, Majda AJ. Strategies for model reduction: comparing different optimal bases. J. Atmos. Sci., 2004; 61: 2306-2317.

7. Majda AJ, Timofeyev I, Vanden-Eijnden E. Systematic strategies for stochastic mode reduction in climate. J. Atmos. Sci., 2003; 60: 1705-1723.

8. Selten F. Baroclinic empirical orthogonal functions as basis functions in an atmospheric model. J. Atmos. Sci., 1997; 54: 2100-2114.

9. Lumley JL. Coherent Structures in Turbulence. in Meyer RE (ed.), Transition and Turbulence, Academic Press, 1981.

10. Aubry N, Holmes P, Lumley JL, et al. The dynamics of coherent structures in the wall region of a turbulent boundary layer. Journal of Fluid Dynamics, 1988; 192: 115-173.

11. Sirovich L. Turbulence and the dynamics of coherent structures: Part I-III. Quarterly of 
Applied Mathematics, 45(1987) 561-590.

12. Roslin RD, Gunzburger MD, Nicolaides R, et al. A self-contained automated methodology for optimal flow control validated for transition delay. AIAA Journal, 1997; 35: 816-824.

13. Ly HV, Tran HT. Proper Orthogonal Decomposition for Flow Calculations and Optimal Control in a Horizontal CVD Reactor. Quarterly of Applied Mathematics, 2002; 60: 631656.

14. Ko J, Kurdila AJ, Redionitis OK, et al. Synthetic Jets, Their Reduced Order Modeling and Applications to Flow Control. AIAA Paper number 99-1000, 37. Aerospace Sciences Meeting \& Exhibit, Reno, 1999.

15. Moin P, Moser RD. Characteristic-eddy decomposition of turbulence in channel. Journal of Fluid Mechanics, 1989; 200: 417-509.

16. Rajaee M, Karlsson SKF, Sirovich L. Low dimensional description of free shear flow coherent structures and their dynamical behavior. Journal of Fluid Mechanics, 1994; 258: 1401-1402.

17. Cao YH, Zhu J, Luo ZD, Navon IM. Reduced order modeling of the upper tropical Pacific ocean model using proper orthogonal decomposition. Computers $\mathscr{G}$ Mathematics with Applications, 2005 (In press).

18. Kunisch K, Volkwein S. Galerkin proper orthogonal decomposition methods for parabolic problems. Numerische Mathematik, 2001; 90: 177-148.

19. Kunisch K, Volkwein S. Galerkin proper orthogonal decomposition methods for a general equation in fluid dynamics. SIAM J. Numer. Anal., 2002; 40(2): 492-515.

20. Kunisch K, Volkwein S. Control of Burgers' equation by a reduced order approach using proper orthogonal decomposition. Journal of Optimization Theory and Applications, 1999; 102: $345-371$.

21. Ahlman D, Södelund F, Jackson J, Kurdila A, Shyy W. Proper orthogonal decomposition for time-dependent lid-driven cavity flows. Numerical Heal Transfer Part B-Fundamentals, 2002; 42(4): 285-306.

22. Cane MA. The response of an equatorial ocean to simple wind stress patterns: Part I. Model formulation and analytical results. J. Mar. Res., 1979; 37: 233-252.

23. Moore DW, Philander SGH. Modeling of the tropical ocean circulation. the Sea, E. D. Goldbrick, Ed., Marine Modeling, Wiley and Sons, 1978; 6: 319-361.

24. Seager R, Zebiak SE, Cane MA. A model of the tropical Pacific sea surface temperature climatology. J. Geophys. Res., 1988; 93: 1265-1280.

25. Yu L, O'Brien JJ. Variational data assimilation for determining the seasonal net surface flux using a tropical Pacific Ocean model. Journal of Physical Oceanography, 1995; 5: 2319-2343.

26. Stricherz J, O'Brien JJ, Legler DM. Atlas of Florida State University Tropical Pacific Winds for TOGA 1966-1985. Florida State University, Tallahassee, FL, 1992.

\section{APPENDIX A}

The proof of (28). First, by expanding all terms of (3) at point $\left(x_{j}, y_{k}, t_{n+1}\right)$ and using Taylor expansion yields

$$
h_{j, k}^{n}=h_{j, k}^{n+1}-\triangle t\left(\frac{\partial h}{\partial t}\right)_{j, k}^{n+1}+\frac{(\triangle t)^{2}}{2 !}\left(\frac{\partial^{2} h}{\partial t^{2}}\right)_{j, k}^{n+1}-\frac{(\triangle t)^{3}}{3 !}\left(\frac{\partial^{3} h}{\partial t^{3}}\right)_{j, k}^{n+1}+\cdots
$$




$$
\begin{aligned}
u_{j+\frac{1}{2}, k}^{n+1}-u_{j-\frac{1}{2}, k}^{n+1}=u_{j+\frac{1}{2}, k}^{n+1}-u_{j, k}^{n+1}+u_{j, k}^{n+1}-u_{j-\frac{1}{2}, k}^{n+1} & \frac{\triangle x}{2}\left(\frac{\partial u}{\partial x}\right)_{j, k}^{n+1}+\frac{1}{2 !}\left(\frac{\triangle x}{2}\right)^{2}\left(\frac{\partial^{2} u}{\partial x^{2}}\right)_{j, k}^{n+1}+\frac{1}{3 !}\left(\frac{\triangle x}{2}\right)^{3}\left(\frac{\partial^{3} u}{\partial x^{3}}\right)_{j, k}^{n+1}+\cdots \\
& +\frac{\triangle x}{2}\left(\frac{\partial u}{\partial x}\right)_{j, k}^{n+1}-\frac{1}{2 !}\left(\frac{\triangle x}{2}\right)^{2}\left(\frac{\partial^{2} u}{\partial x^{2}}\right)_{j, k}^{n+1}+\frac{1}{3 !}\left(\frac{\triangle x}{2}\right)^{3}\left(\frac{\partial^{3} u}{\partial x^{3}}\right)_{j, k}^{n+1}-\cdots \\
= & \triangle x\left(\frac{\partial u}{\partial x}\right)_{j, k}^{n+1}+\frac{(\triangle x)^{3}}{24}\left(\frac{\partial^{3} u}{\partial x^{3}}\right)_{j, k}^{n+1}+\cdots, \\
v_{j, k+\frac{1}{2}}^{n+1}-v_{j, k-\frac{1}{2}}^{n+1}=v_{j, k+\frac{1}{2}}-v_{j, k}^{n+1}+v_{j, k}^{n+1}-v_{j, k-\frac{1}{2}}^{n+1} & \frac{\triangle y}{2}\left(\frac{\partial v}{\partial y}\right)_{j, k}^{n+1}+\frac{1}{2 !}\left(\frac{\triangle y}{2}\right)^{2}\left(\frac{\partial^{2} v}{\partial y^{2}}\right)_{j, k}^{n+1}+\frac{1}{3 !}\left(\frac{\triangle y}{2}\right)^{3}\left(\frac{\partial^{3} v}{\partial y^{3}}\right)_{j, k}^{n+1}+\cdots \\
& +\frac{\triangle y}{2}\left(\frac{\partial v}{\partial y}\right)_{j, k}^{n+1}-\frac{1}{2 !}\left(\frac{\triangle y}{2}\right)^{2}\left(\frac{\partial^{2} v}{\partial y^{2}}\right)_{j, k}^{n+1}+\frac{1}{3 !}\left(\frac{\triangle y}{2}\right)^{3}\left(\frac{\partial^{3} v}{\partial y^{3}}\right)_{j, k}^{n+1}-\cdots \\
= & \triangle y\left(\frac{\partial v}{\partial y}\right)_{j, k}^{n+1}+\frac{(\triangle y)^{3}}{24}\left(\frac{\partial^{3} v}{\partial y^{3}}\right)_{j, k}^{n+1}+\cdots
\end{aligned}
$$

Inserting (30)-(32) into (3) yields

$$
\begin{aligned}
& \left(\frac{\partial h}{\partial t}\right)_{j, k}^{n+1}+H\left[\left(\frac{\partial u}{\partial x}\right)_{j, k}^{n+1}+\left(\frac{\partial v}{\partial y}\right)_{j, k}^{n+1}\right] \\
& =\frac{\triangle t}{2 !}\left(\frac{\partial^{2} h}{\partial t^{2}}\right)_{j, k}^{n+1}-\frac{(\triangle x)^{2}}{24}\left(\frac{\partial^{3} u}{\partial x^{3}}\right)_{j, k}^{n+1}-\frac{(\triangle y)^{2}}{24}\left(\frac{\partial^{3} v}{\partial y^{3}}\right)_{j, k}^{n+1}+\cdots .
\end{aligned}
$$

Therefore, the truncation error that (3) approximates to $\frac{\partial h}{\partial t}+H\left(\frac{\partial^{2} h}{\partial t^{2}}+\frac{\partial^{3} v}{\partial y^{3}}\right)=0$ is

$$
T E_{1}=O\left(\triangle t,(\triangle x)^{2},(\triangle y)^{2}\right)
$$

Second, by expanding all terms of $(5)$ at point $\left(x_{j+\frac{1}{2}}, y_{k}, t_{n}\right)$ using Taylor expansion yields

$$
\begin{aligned}
& u_{j+\frac{1}{2}, k}^{n+1}=u_{j+\frac{1}{2}, k}^{n}+\triangle t\left(\frac{\partial u}{\partial t}\right)_{j+\frac{1}{2}, k}^{n}+\frac{(\triangle t)^{2}}{2 !}\left(\frac{\partial^{2} u}{\partial t^{2}}\right)_{j+\frac{1}{2}, k}^{n}+\frac{(\triangle t)^{3}}{3 !}\left(\frac{\partial^{3} u}{\partial t^{3}}\right)_{j+\frac{1}{2}, k}^{n}+\cdots \\
& h_{j+1, k}^{n}-h_{j, k}^{n}=h_{j+1, k}^{n}-h_{j+\frac{1}{2}, k}^{n}+h_{j+\frac{1}{2}, k}^{n}-h_{j, k}^{n} \\
& =\left(\frac{\triangle x}{2}\right)\left(\frac{\partial h}{\partial x}\right)_{j+\frac{1}{2}, k}^{n}+\frac{1}{2 !}\left(\frac{\triangle x}{2}\right)^{2}\left(\frac{\partial^{2} h}{\partial x^{2}}\right)_{j+\frac{1}{2}, k}^{n}+\frac{1}{3 !}\left(\frac{\triangle x}{2}\right)^{3}\left(\frac{\partial^{3} h}{\partial x^{3}}\right)_{j+\frac{1}{2}, k}^{n}+\cdots \\
& +\left(\frac{\triangle x}{2}\right)\left(\frac{\partial h}{\partial x}\right)_{j+\frac{1}{2}, k}^{n}-\frac{1}{2 !}\left(\frac{\triangle x}{2}\right)^{2}\left(\frac{\partial^{2} h}{\partial x^{2}}\right)_{j+\frac{1}{2}, k}^{n}+\frac{1}{3 !}\left(\frac{\triangle x}{2}\right)^{3}\left(\frac{\partial^{3} h}{\partial x^{3}}\right)_{j+\frac{1}{2}, k}^{n}+\cdots \\
& =\triangle x\left(\frac{\partial h}{\partial x}\right)_{j+\frac{1}{2}, k}^{n}+\frac{(\triangle x)^{3}}{24}\left(\frac{\partial^{3} h}{\partial x^{3}}\right)_{j+\frac{1}{2}, k}^{n}+\cdots
\end{aligned}
$$




$$
\begin{aligned}
& u_{j+\frac{1}{2}, k-1}-2 u_{j+\frac{1}{2}, k}+u_{j+\frac{1}{2}, k+1}=\left[u_{j+\frac{1}{2}, k-1}-u_{j+\frac{1}{2}, k}\right]+\left[u_{j+\frac{1}{2}, k+1}-u_{j+\frac{1}{2}, k}\right] \\
& =\left[-\triangle y\left(\frac{\partial u}{\partial y}\right)_{j+\frac{1}{2}, k}^{n}+\frac{(\triangle y)^{2}}{2 !}\left(\frac{\partial^{2} u}{\partial y^{2}}\right)_{j+\frac{1}{2}, k}^{n}-\frac{(\triangle y)^{3}}{3 !}\left(\frac{\partial^{3} u}{\partial y^{3}}\right)_{j+\frac{1}{2}, k}^{n}\right. \\
& \left.+\frac{(\triangle y)^{4}}{4 !}\left(\frac{\partial^{4} u}{\partial y^{4}}\right)_{j+\frac{1}{2}, k}^{n}+\cdots\right]+\left[\triangle y\left(\frac{\partial u}{\partial y}\right)_{j+\frac{1}{2}, k}^{n}+\frac{(\triangle y)^{2}}{2 !}\left(\frac{\partial^{2} u}{\partial y^{2}}\right)_{j+\frac{1}{2}, k}^{n}\right. \\
& \left.+\frac{(\triangle y)^{3}}{3 !}\left(\frac{\partial^{3} u}{\partial y^{3}}\right)_{j+\frac{1}{2}, k}^{n}+\frac{(\triangle y)^{4}}{4 !}\left(\frac{\partial^{4} u}{\partial y^{4}}\right)_{j+\frac{1}{2}, k}^{n}+\cdots\right] \\
& =(\triangle y)^{2}\left(\frac{\partial^{2} u}{\partial y^{2}}\right)_{j+\frac{1}{2}, k}^{n}+\frac{(\triangle y)^{4}}{12}\left(\frac{\partial^{4} u}{\partial y^{4}}\right)_{j+\frac{1}{2}, k}^{n}+\cdots, \\
& u_{j-\frac{1}{2}, k}-2 u_{j+\frac{1}{2}, k}+u_{j+\frac{3}{2}, k}=\left[u_{j-\frac{1}{2}, k}-u_{j+\frac{1}{2}, k}\right]+\left[u_{j+\frac{3}{2}, k}-u_{j+\frac{1}{2}, k}\right] \\
& =\left[-\triangle x\left(\frac{\partial u}{\partial x}\right)_{j+\frac{1}{2}, k}^{n}+\frac{(\triangle x)^{2}}{2 !}\left(\frac{\partial^{2} u}{\partial x^{2}}\right)_{j+\frac{1}{2}, k}^{n}-\frac{(\triangle x)^{3}}{3 !}\left(\frac{\partial^{3} u}{\partial x^{3}}\right)_{j+\frac{1}{2}, k}^{n}\right. \\
& \left.+\frac{(\triangle x)^{4}}{4 !}\left(\frac{\partial^{4} u}{\partial x^{4}}\right)_{j+\frac{1}{2}, k}^{n}+\cdots\right]+\left[\triangle x\left(\frac{\partial u}{\partial x}\right)_{j+\frac{1}{2}, k}^{n}+\frac{(\triangle x)^{2}}{2 !}\left(\frac{\partial^{2} u}{\partial x^{2}}\right)_{j+\frac{1}{2}, k}^{n}\right. \\
& \left.+\frac{(\triangle x)^{3}}{3 !}\left(\frac{\partial^{3} u}{\partial x^{3}}\right)_{j+\frac{1}{2}, k}^{n}+\frac{(\triangle x)^{4}}{4 !}\left(\frac{\partial^{4} u}{\partial x^{4}}\right)_{j+\frac{1}{2}, k}^{n}+\cdots\right] \\
& =(\triangle x)^{2}\left(\frac{\partial^{2} u}{\partial x^{2}}\right)_{j+\frac{1}{2}, k}^{n}+\frac{(\triangle x)^{4}}{12}\left(\frac{\partial^{4} u}{\partial x^{4}}\right)_{j+\frac{1}{2}, k}^{n}+\cdots,
\end{aligned}
$$

Inserting (35)-(38) into (5) yields

$$
\begin{aligned}
& \left(\frac{\partial u}{\partial t}\right)_{j+\frac{1}{2}, k}^{n}-\left(f v+\frac{\tau^{x}}{\rho_{0} H}\right)_{j+\frac{1}{2}, k}^{n}+g^{\prime}\left(\frac{\partial h}{\partial x}\right)_{j+\frac{1}{2}, k}^{n}+A\left[\left(\frac{\partial^{2} u}{\partial x^{2}}\right)_{j+\frac{1}{2}, k}^{n}+\left(\frac{\partial^{2} u}{\partial y^{2}}\right)_{j+\frac{1}{2}, k}^{n}\right] \\
& =-\frac{\triangle t}{2 !}\left(\frac{\partial^{2} h}{\partial t^{2}}\right)_{j, k}^{n+1}-g^{\prime} \frac{(\triangle x)^{2}}{24}\left(\frac{\partial^{3} h}{\partial x^{3}}\right)_{j+\frac{1}{2}, k}^{n} \\
& +A \frac{(\triangle x)^{2}}{12}\left(\frac{\partial^{4} u}{\partial x^{4}}\right)_{j, k}^{n+1}+A \frac{(\triangle y)^{2}}{12}\left(\frac{\partial^{4} v}{\partial y^{4}}\right)_{j, k}^{n+1}+\cdots .
\end{aligned}
$$

Therefore, the truncation error that (5) approximates to (4) is

$$
T E_{2}=O\left(\triangle t,(\triangle x)^{2},(\triangle y)^{2}\right) .
$$

Next, using the same approach as in (40), the truncation error that (8) approximates to (7) is given by

$$
T E_{3}=O\left(\triangle t,(\triangle x)^{2},(\triangle y)^{2}\right) .
$$

Since (10) is developed from (3), (5), and (8), the error of numerical solutions $\left(u_{j+\frac{1}{2}, k}^{n}, v_{j, k+\frac{1}{2}}^{n}\right.$, $\left.h_{j, k}^{n}\right)(1 \leq n \leq N, 0 \leq j \leq J, 0 \leq k \leq K)$ for Problem (I) obtained by (5), (8), and (10)

$$
\begin{aligned}
\left|E_{n}\left(u_{j+\frac{1}{2}, k}^{n}, v_{j, k+\frac{1}{2}}^{n}, h_{j, k}^{n}\right)\right|= & \|\left(u\left(x_{j+\frac{1}{2}}, y_{k}, t_{n}\right), v\left(x_{j}, y_{k+\frac{1}{2}}, t_{n}\right), h\left(x, y_{k}, t_{n}\right)\right) \\
& -\left(u_{j+\frac{1}{2}, k}^{n}, v_{j, k+\frac{1}{2}}^{n}, h_{j, k}^{n}\right) \|_{2}=O\left(\Delta t, \Delta x^{2}, \Delta y^{2}\right),
\end{aligned}
$$

which completes the proof of (28). 